\title{
Penetration of the Optic Nerve and Falciform Ligament by an Internal Carotid Artery-Ophthalmic Artery Aneurysm: Case Reoport
}

\author{
Yasushi TAKAGI $^{1}$ and Susumu MIYAMOTO ${ }^{1}$ \\ ${ }^{1}$ Department of Neurosurgery, Kyoto University Graduate School of Medicine, Kyoto, Kyoto
}

\begin{abstract}
We report a case of an internal carotid artery (ICA)-ophthalmic artery aneurysm with penetration of the optic nerve. In addition, this case penetrated the falciform ligament, which severely disturbed optic canal unroofing during surgery. This is the first reported case in which penetration of the optic nerve and falciform ligament has been shown. To remove the anterior clinoid process in this case, the ultrasonic bone curette was a useful tool.
\end{abstract}

Key words: internal carotid artery (ICA)-ophthalmic artery aneurysm, penetration, optic nerve

\section{Introduction}

Internal carotid artery (ICA)-ophthalmic artery aneurysms have a small proportion of intracranial aneurysms. They constitute $0.3 \%$ to $1 \%$ of intracranial aneurysms and $0.9 \%$ to $6.5 \%$ of aneurysms of the ICA. ${ }^{1)}$ Yasargil ${ }^{2)}$ reported that $18 \%$ of patients with aneurysms of the ophthalmic segment presented with visual symptoms. It is rare, however, for the aneurysm to penetrate or physically split the optic apparatus. There have been only a limited number of case reports illustrating optic nerve penetration by an ophthalmic artery aneurysm. ${ }^{3-6)}$ We report a case of an ICA-ophthalmic artery aneurysm with penetration of the optic nerve. In addition, our case penetrated the falciform ligament, which severely disturbed optic canal unroofing during surgery. This is the first reported case in which penetration of the optic nerve and falciform ligament has been shown.

\section{Case Report}

The patient was a 62-year-old woman with no significant medical history. She had presented with blurred vision in her left eye for 1 month. Magnetic resonance imaging (MRI) scans demonstrated a less than $1 \mathrm{~cm}$ cerebral aneurysm arising from the wall of the left ICA and existed nearby left optic nerve (Fig. 1A, C). Cerebral angiograms confirmed that the ICA aneurysm originated from the ophthalmic segment, just distal to the origin

Received December 6, 2012; Accepted January 28, 2013 of the ophthalmic artery, and measured $7 \mathrm{~mm}$ (height) with a $3 \mathrm{~mm}$ neck (Fig. 1B). After informed consent for surgical treatment, the patient received a left pterional craniotomy after exposure of left ICA in the neck. The dissection was continued to the right optic nerve-chiasm junction. A reddish part in the falciform ligament covered the optic nerve (Fig. 2A, arrow). After removing the falciform ligament for optic canal unroofing, the tip of the aneurysm penetrated the optic nerve (Fig. 2B, arrow). The aneurysm also penetrated the optic sheath and eroded the bone edge at the entrance of the optic canal. It was immediately apparent that it would be necessary to drill the anterior clinoid process and optic canal to uncover the proximal neck. A part of the anterior clinoid process and the roof of the optic canal were removed carefully by an ultrasonic bone curette (Fig. 2C). The direction of the bone curette was used parallel to the aneurysmal dome not to injure its wall. After that thinned bone was removed by micro dissector. This scalpel was very useful because the surface of the scalpel was moved towards the opposite side of the dome of the aneurysm. The aneurysmal neck was confirmed at the origin of ophthalmic artery and the dome was detached from the optic nerve. The patient underwent successful clipping of the aneurysm, with application of a Yasargil clip under the left optic nerve (Fig. 2D). Postoperative computed tomography (CT) angiography demonstrated complete obliteration of the aneurysm. The patient's postoperative course was uneventful, and her lower quadrantanopsia and visual acuity remained unchanged at the 3 -month follow-up examination. 

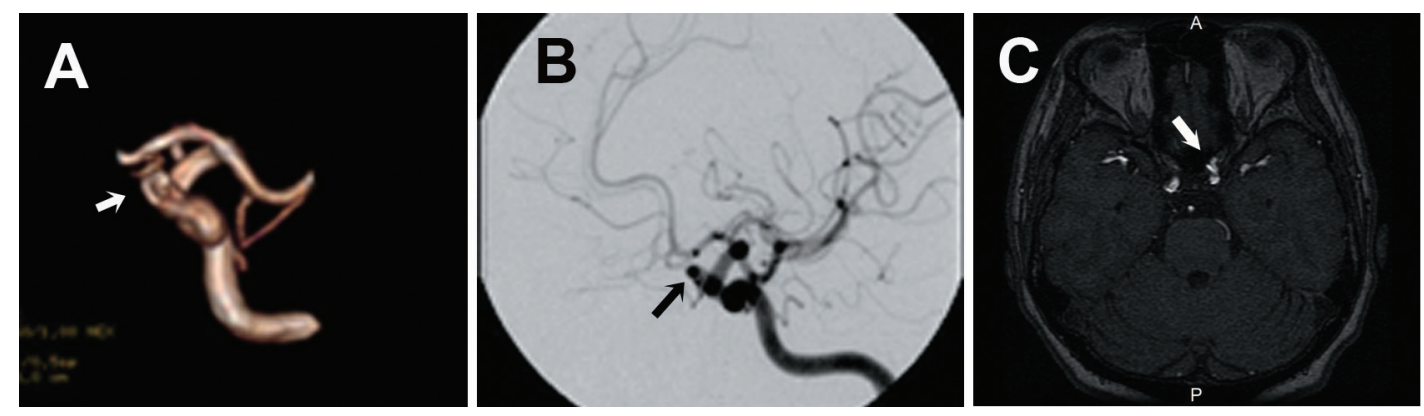

Fig. 1 Preoperative images. A: Magnetic resonance (MR) angiography indicated a left internal carotid artery (ICA) aneurysm (arrow). B: Left internal carotid angiogram disclosed an ICA-ophthalmic artery aneurysm (arrow). C: Original MR angiography images indicated the aneurysm close to the optic nerve (arrow).
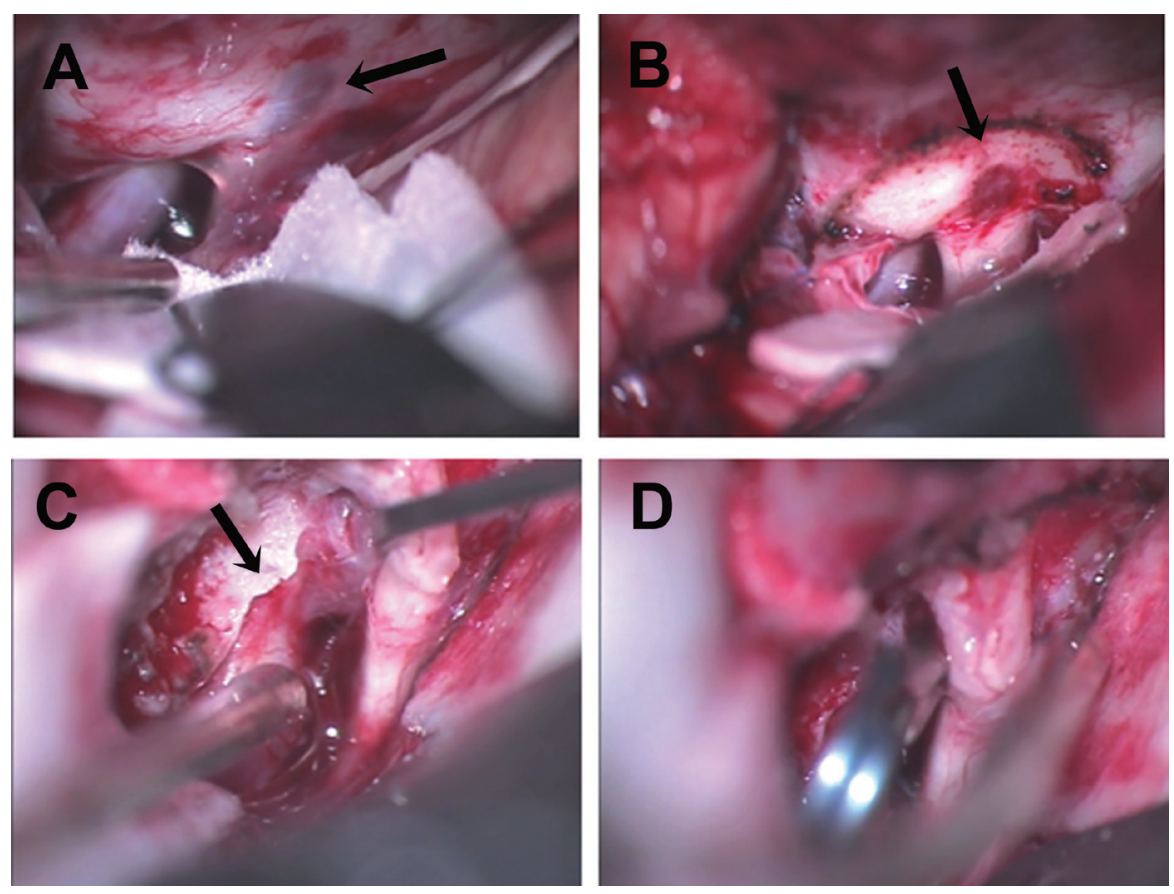

Fig. 2 Intraoperative view. A: The aneurysm was through the falciform ligament (arrow). B: After removal of the falciform ligament, the aneurysm penetrated the optic nerve and eroded the roof of the optic canal (arrow). C: After removal of the anterior clinoid process, the neck of the aneurysm was confirmed (arrow). D: After clipping, the aneurysm shrank.

\section{Discussion}

Eight cases of penetration of the optic apparatus by an intracranial aneurysm have been reported., ${ }^{1,3-9)}$ Four were ICA-ophthalmic artery aneurysms, three were anterior communicating artery aneurysms, and one was an ICA anterior wall aneurysm.

Our case is noteworthy in two respects. First, the aneurysm penetrated not only the optic nerve but also the falciform ligament and eroded the bony structure. This is the first reported case of falciform ligament penetration. The aneurysm was confirmed to penetrate through the falciform ligament. Second, for surgery it is necessary to remove the anterior clinoid process for neck clipping. Removal of the anterior clinoid process and the optic canal unroofing was extremely dangerous in this case.
The ultrasonic bone curette was very useful to avoid accidental injury of the aneurysm.

Regarding the mechanism of optic nerve penetration in the unruptured aneurysm, in a previous report, it occurred with a rapidly expanding unruptured aneurysm. ${ }^{4)}$ There has been only one case report describing this mechanism. Fujita et al. (2002) ${ }^{4)}$ described the potentially aggressive behavior of unruptured aneurysms of the ophthalmic segment and hypothesized that a rapid increase in aneurysm size allowed the aneurysm to push through the tightly bound fibers of the optic nerve. In another report by Jea et al. $(2003)^{5)}$ the aneurysm grew slowly with time, in a direction dictated by hemodynamic flow factors, so that the vector of expansion was directed towards the part of the optic nerve that eventually became the split. It is difficult to conclusively prove the exact mechanism of optic nerve penetration in 
our case. About the site of aneurysm neck, we confirmed an ophthalmic artery during surgical procedure. But an anomalous artery sometimes originates from the ICA at the branching of the ophthalmic artery. The anomaly might be explained by persistence to the time of full development of a vascular anastomotic loop normally present only in the embryo. One possibility is the transitory communication between primitive dorsal and ventral ophthalmic arteries. The other is a potential anastomosis between the primitive olfactory and maxillary arteries. These discussions were described by Kanamaru et al (2007). ${ }^{1)}$

As for use of the ultrasonic bone curette, after identifying the proximal orifice of the optic canal extradurally, bone resection could be initiated from the proximal edge of the canal roof. ${ }^{10)}$ This is easily accomplished by placing the tip of the ultrasonic bone curette on the edge of the canal roof and applying the ultrasonic energy in short bursts. ${ }^{10)}$ The bone resection proceeds in a proximal-todistal direction. In addition, the ultrasonic bone curette has another technical advantage. During the unroofing procedure, we were always able to identify the edge of the bone. This allowed us to resect only as much of the bone over the canal as was necessary, always knowing how much of the canal had been opened. ${ }^{10)}$ Drilling of the optic canal, however, poses its own risks to the optic nerve. Any inadvertent contact of the rapidly rotating drill bit with the optic sheath or the frontal dura will create a tear and may even damage the nerve or the frontal lobe. Especially in our case, it was extremely difficult to remove the anterior clinoid process because of the nearby aneurysm dome. Thus the ultrasonic bone scalpel was especially useful in our case.

As for preoperative diagnosis, to confirm the relationship between aneurysm and optic nerve, coronal thin slice MRI is necessary. Furthermore in the case with preoperative severe visual field loss or loss of visual acuity, intravascular should be considered. During neck clipping of paraclinoid aneurysms, we emphasized that exposure of cervical ICA is necessary.

In summary, we describe for the first time a case involving penetration of the optic nerve and falciform ligament. In addition, to remove the anterior clinoid process in this case, the ultrasonic bone curette was a useful tool.

\section{Conflicts of Interest Disclosure}

All authors have no conflicts of interest. In addition, authors who are members of The Japan Neurosurgical
Society (JNS) state that all authors have registered online Self-reported COI Disclosure Statement Forms through the website for JNS members.

\section{References}

1) Kanamaru K, Ishida F, Taki W: Splitting and penetration of the optic nerve by an aneurysm arising from the anterior wall of internal carotid artery: case report. J Neurol Neurosurg Psychiatry 71: 525-527, 2001

2) Yasargil MG: Carotid-ophthalmic aneurysms, in Microneurosurgery: Clinical Considerations, Surgery of the Intracranial Aneurysms and Results, vol II. Stuttgart, Georg Thieme, 1984, pp 43-55

3) Beatty RA: Splitting of the optic nerve by a carotid-ophthalmic artery aneurysm. Case report. J Neurosurg 65: 560-562, 1986

4) Fujita A, Tamaki N, Yasuo K, Nagashima T, Ehara K: Complete penetration of the optic chiasm by an unruptured aneurysm of the ophthalmic segment: case report. Surg Neurol 57: 130-134, 2002

5) Jea A, Başkaya MK, Morcos JJ: Penetration of the optic nerve by an internal carotid artery-ophthalmic artery aneurysm: case report and literature review. Neurosurgery 53: 996-999; discussion 999-1000, 2003

6) Wang YY, Thani NB, Han TF: Optic nerve penetration by a carotico-ophthalmic artery aneurysm. J Clin Neurosci 17: 931-933, 2010

7) Date I, Akioka T, Ohmoto T: Penetration of the optic chiasm by a ruptured anterior communicating artery aneurysm. Case report. J Neurosurg 87: 324-326, 1997

8) Joo SP, Kim TS: Splitting of the optic nerve associated with ruptured anterior communicating artery aneurysm. Neurol Med Chir (Tokyo) 47: 501-502, 2007

9) Lee SH, Koh JS, Lee CY, Kwon GY, Ryu CW: Penetration and splitting of the optic apparatus by intrasaccular coils within an anterior communicating artery aneurysm. ClinNeurol Neurosurg 113: 578-581, 2011

10) Chang HS, Joko M, Song JS, Ito $K$, Inoue $T$, Nakagawa $H$ : Ultrasonic bone curettage for optic canal unroofing and anterior clinoidectomy. Technical note. J Neurosurg 104: 621-624, 2006

Address reprint requests to: Yasushi Takagi, MD, PhD, Department of Neurosurgery, Graduate School of Medicine, Kyoto University, 54 Kawahara-cho, Shogoin, Sakyo, Kyoto, Kyoto 606-8508, Japan.

e-mail: ytakagi@kuhp.kyoto-u.ac.jp 